\title{
DIE -GRIECHISCHEN
}

\section{CHRISTLICHEN SCHRIFTSTELLER \\ DER}

\section{ERSTEN DREI JAHRHUNDERTE}

HERAUSGEGEBEN VON DER KIRCHENVÄTER-COMMISSION

DER KÖNIGL. PREUSSISCHEN AKADEMIE DER WISSENSCHAFTEN

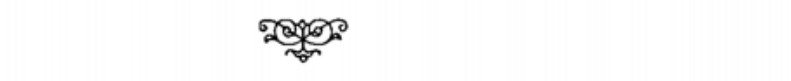

\section{CLEMENS ALEXANDRINUS}

DRITTER BAND

LEIPZIG

J. C. HINRICHS'SCHE BUCHHANDLUNG

1909 
IN DER REIHENFOLGE DES ERSCHEINENS BAND 17

Druck von A ugust Pries in Leipzig 


$$
\text { - }
$$





\title{
CLEMENS ALEXANDRINUS
}

DRITTER BAND

\author{
STROMATA BUCH VII UND VIII \\ EXCERPTA EX THEODOTO - ECLOGAE PROPHETICAE \\ QUIS DIVES SALVETUR - FRAGMENTE
}

\author{
HERAUSGEGEBEN \\ IM AUFTRAGE DER KIRCHENVÄTER-COMMISSION \\ DER KÖNIGL. PREUSSISCHEN AKADEMIE DER WISSENSCHAFTEN \\ roN \\ DR. OTTO STÄHLIN \\ PROFESSOR AN DER UNIVERSITÄT WÜRZBURG
}

MIT DREI HANDSCHRIFTENPROBEN IN LICHTDRUCK

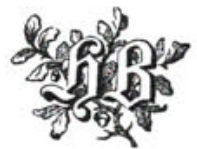

LEIPZIG

J. C. HINRICHS'SCHE BUCHHANDLUNG 1909 
